\title{
Towards an evidence based alcohol policy
}

\author{
Ranil Abeyasinghe
}

\section{Summary}

Alcohol abuse is a major problem in Sri Lanka. Alcohol policies cannot be based on religious views, political expediency or tax revenue. Pitfalls in following policies followed in developed societies without regard to the local conditions abound. A sufficient body of evidence based knowledge exists today in the medical literature to help formulate a rational alcohol policy that suits Sri Lanka. The public and private sector agencies along with the medical profession should play an active role in formulating and enacting such a policy. The need to promote local research into alcohol related problems is also emphasized.

SL J Psychiatry 2011; 2 (1):1-2

Recently a member of the legislative assembly in Pakistan introduced a motion to set aside the total ban on alcohol enacted in 1977. He stated that in the absence of alcohol, Pakistani youth were turning to dangerous substances such as heroin. Pakistan today has an estimated population of 500,000 chronic heroin users, one of the highest use rates per population in the world (1).The emergence of this trend of heroin use has been blamed by media and politicians alike on the ban on all alcohol sales in 1977 by the then President of the country, Zia ul Haq. The ban on alcohol originated in politics and the moves to lift it now seems to have political undertones as well. History bears evidence as to how other countries have learned bitter lessons from such top down alcohol policies. The Mafia emerged in the US after the government banned alcohol sales in the 1920s (2). Czar Nicholas II in imperial Russia banned the manufacture and sale of vodka and had to contend with a revolution in 1914 in which the masses ransacked his alcohol supplies!

Sri Lanka too has seen a mixed bag of alcohol policies that were politically driven and not based on evidence. For example, when the move to ban advertising and sports sponsorship for alcohol and tobacco was enforced in 2007, it was accepted wholeheartedly by the medical community.

However, after a sharp drop in the sales of alcohol in 2007 following the ban on advertising, there has been a gradual but sharp increase of alcohol sales in the country (Fig.1). It may be argued that the economic downturn during the civil unrest in 2007-09 caused the sharp decline and that the subsequent increase of sales is related to the post-war economic recovery. For whatever reason, alcohol sales have risen despite an advertising ban.

\section{Making alcohol policy evidence based}

Just as much as the practice of medicine is increasingly becoming evidence based, so must health related policies. It is time that the medical profession brought to bear its considerable weight to formulate a rational alcohol policy that is evidence based. Such evidence exists at three different levels.

At the highest level are findings that have come from repeatedly reproduced research. One such finding is that of the relationship between the rates of cirrhosis related mortality and alcohol abuse in a country (3). In 2005, the
Census and Statistics Department in Sri Lanka reported a cirrhosis mortality rate of 33.4 per 100,000 males, among the highest in the world which compares with 14.1 in the United Kingdom and 28.14 in France (4). A second factor is that the increasing mortality and morbidity of unhealthy drinking patterns far outweigh the medical benefits of social drinking $(5,6,7)$. Athird is that alcohol abuse is a major cause of poverty in Sri Lanka (8). A fourth reason is that those hazardous drinkers when educated about the benefits of reducing alcohol consumption, particularly by doctors, cut down on their drinking (9). Routine questions about alcohol consumption by doctors and brief intervention have shown to help.

A second tier of evidence exists that could help to formulate a rational alcohol policy. It is proposed to rate such evidence as 'second tier' due to the existence offindings that are equivocal. For instance, there is evidence to suggest that by raising the price of alcohol a society could restrict the availability of alcohol (10). However, this does not seem to extend as far as physical restriction of alcohol as was shown to be effective in the United States during the prohibition in 1920s. Neither physical restriction nor price increase seems to matter in countries where an indigenous illicit alcohol industry exists. In Sri Lanka, increases in the prices of legal alcohol led to an increase in illicit alcohol consumption and a surge of alcohol related disorders (11). The much hyped mode of preventing alcohol related disorders via the restriction of advertising has not had the desired effect in Sri Lanka. There is also evidence that educating the public about alcohol through moral and religious elements may only encourage adolescents to drink (12). Where people were given information regarding benefits of moderate drinking, there was a marked drop in alcohol consumption in a Sri Lankan village (13). Another area that is worthy of consideration in a future alcohol policy is the harm reduction concept. Harm reduction is based on the prevention of secondary and tertiary complications arising from heavy drinking, rather than achieving abstinence. This approach, familiar to psychiatrists treating disorders such as schizophrenia, has much value $(14,15)$. Towards this end, setting up of alcohol treatment centres has helped (16). However such an approach requires a tightly knit alcohol policy.

Another area that an alcohol policy should have an impact is the drinking culture. This determines the type of beverage, 


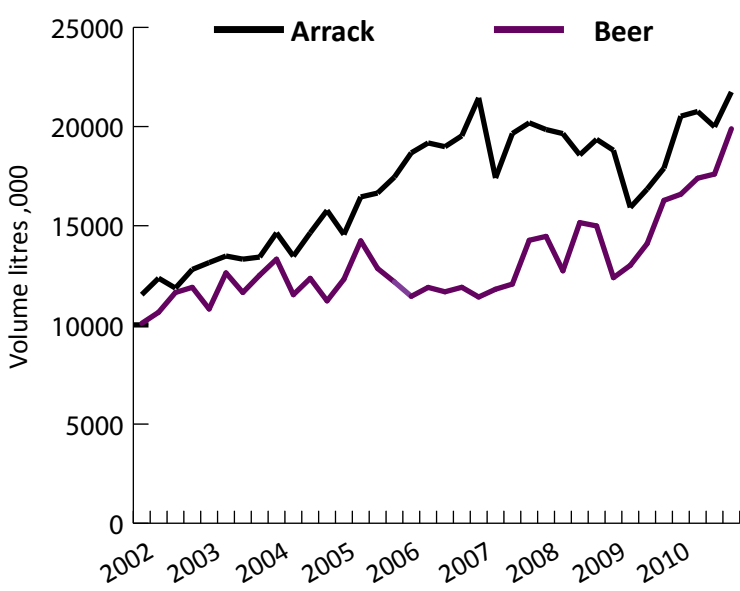

Fig. 1 Arrack and beer sales in Sri Lanka 2002-2010

Source:Department of Excise, Sri lanka

the amounts consumed and even the expected outcome of drinking. Where inebriation is the expectation of a drinking culture its immediate and long term deleterious results ensue. A future alcohol policy must address this issue of the drinking culture where people consume alcohol for the purpose of intoxication ('drinking to get drunk').

The third tier of evidence is where the adopted alcohol policies have either not been tested or when tested have not produced the desirable effects. Excise holidays, server training, warning labels are some such measures where there is little research or the findings are unconvincing, despite such seemingly well intentioned measures.

\section{Political will must translate into an evidence based alcohol policy}

Political will to reduce alcohol harm should translate into legislation where an integrated approach is developed in all public and private sector agencies towards reducing alcohol consumption. This has to extend from the departments of health, police, excise and education to the ministries of public administration and social services. If the police were to deal with public drunkenness with the same zeal that they displayed when dealing with public smoking, there would be less drinking to the level of intoxication. If the police enforced drink driving laws strictly there would be less drinking. The same would be true if the government and the private sector had a policy of performing regular screening for alcohol and drug abuse for employees. Government welfare recipients too should be subject to such routine screening. It is such an overall strategy to eliminate alcohol abuse rather than the more trendy 'mathata thitha' (literally, a full stop to alcohol) program that will one day reduce widely prevalent alcohol abuse in Sri Lanka.

\section{Need for more locally relevant research}

As outlined above, second and third tier evidence essential to formulate a national alcohol policy requires research in the local culture. This is either due to the weakly positive or negative findings in other countries or due to the need to reproduce such results in the local culture. Thus a future national alcohol policy should either set up a research arm or set apart funds for those doing such alcohol related research. Such funds could appropriately come from alcohol taxation.

\section{Declaration of interest}

None
Ranil Abeyasinghe, MBBS, MD(Psych), MRCPsych, Head \& Senior Lecturer, Department of Psychiatry, Faculty of Medicine, University of Peradeniya, Sri Lanka and Consultant Psychiatrist, Teaching Hospital, Peradeniya, Sri Lanka

E mail: ranil52@yahoo.com

\section{References}

1. Illicit Drug Trends in Pakistan. UNDOC report, 2008 Available at: http://www.unodc.org/documents/regional/ central-asia/Illicit\%20Drug\%20Trends\%20Report_ Pakistan_rev1.pdf

2. Cervais CH. The Rum Runner, A prohibition scrap book, , Thornhill: Firefly Book,1980

3. Ramstedt M. Per capita consumption and liver cirrhosis mortality in 14 European countries. Addiction 2001; 960 (1): 19-33.

4. Leon $\mathrm{AD}$, McCambridge J. Liver cirrhosis mortality rates in Britain from 1950 to 2002:an analysis of routine data. Lancet 2006; 367: 52-56

5. Marmot M, Brunner E. Alcohol and cardiovascular disease: the status of the U shaped curve. BMJ 1991;303:565-8.

6. Doll R, Peto R, Hall E, Wheatley K, Gray R. Mortality in relation to consumption of alcohol: 13 years' obeservations on male British doctors BMJ 1994; 309: 911

7. Brien SE, Ronksley PE, Turner BJ, Mukamal KJ, Ghali WA. Effect of alcohol on biological makers associated with the risk of coronary heart disease: systematic review and meta analysis of interventional studies BMJ. 2011;342:d636. doi: 10.1136/bmj.d636.

8. De Silva V, Samarasinghe D, Hanwella R. Association between concurrent alcohol and tobacco use and poverty. Drug Alcohol Rev 2011;30(1):69-73.

9. Moyer A, Finney JW. Brief interventions for alcohol problems: a meta-analytic review of controlled investigations in treatment-seeking and non-treatment-seeking population. Addiction 2002;97(3): 279-292.

10. Wagenaar AC, Salois MJ, Komro KA. Effects of beverage alcohol price and tax levels on drinking: a meta-analysis of 1003 estimates from 112 studies. Addiction 2009; 104 (2):179-190.

11. Abeyasinghe R. Illicit Alcohol. Illicit drinking culture in Colombo. Vijitha Yapa. 2001

12. Bock EW, Cochran JK, Beeghley L. Moral messages: The relative influence of denomination the religiosity-alcohol relationship. Sociological Quarterly 2008; 28 (1) 89-103.

13. Abeyasinghe R, Dawson A, Siriwardana P, Dias S, Tennakoon S. Randomized controlled trial of a community based alcohol education programme to change the drinking pattern in rural Sri Lanka. College of Psychiatry Annual Sessions 2010

14. Marlett $\mathrm{G}$, Witkiewitz $\mathrm{K}$. Harm reduction approaches to alcohol use, health promotion, prevention and treatment. Addictive Behaviors 2002; 27;867-886.

15. RitterA, Cameron J.Areview of the efficacy and effectiveness of harm reduction strategies for alcohol, tobacco and illicit drugs. Drug Alcohol Rev 2006; 25(6): 611-24.

16. Mann R, Smart RG, Anglin L, Rush BR. Are Decreases in Liver Cirrhosis Rates a Result of Increased Treatment for Alcoholism? British Journal of Addiction 1988; 83 (6): 683-688. 2010) 